\title{
Treatment of steroid-dependent rectal sparing ulcerative colitis associated with severe autoimmune hemolytic anemia with oral mycophenolate mofetil-a case report and review of literature
}

Xin Liu ( $\nabla$ docliuxin@126.com )

the Second Affiliated Hospital of Xi'an Jiaotong University

\section{Sumei Sha}

the Second Affiliated Hospital of Xi'an Jiaotong University

\section{Bin Xu}

the Chenggong Hospital Affiliated to Xiamen University (Central Hospital of the 73th Chinese People's

Liberation Army), Xiamen Fujian Province

Haitao Shi

the Second Affiliated Hospital of Xi'an Jiaotong University

\section{Xiaojing Quan}

the Second Affiliated Hospital of Xi'an Jiaotong University

\section{Fenrong Chen}

the Second Affiliated Hospital of Xi'an Jiaotong University

Jiong Jiang

the Second Affiliated Hospital of Xi'an Jiaotong University

\section{Case Report}

Keywords: ulcerative colitis, autoimmune hemolytic anemia, CMV infect, Coombs test, case report

Posted Date: March 2nd, 2022

DOI: https://doi.org/10.21203/rs.3.rs-1401188/v1

License: (c) (i) This work is licensed under a Creative Commons Attribution 4.0 International License.

Read Full License 


\section{Abstract}

Background: Patients with ulcerative colitis (UC) may experience various type of anemia, in which the most common reasons are dystrophic anemia and hemorrhagic anemia. Autoimmune hemolytic anemia (AlHA) associated with UC is seldom seen, especially Coombs-negative AlHA.

Case presentation: Here we report a case of refractory anemia that cannot be explained by UC disease activity in a young male. The patient was infected with cytomegalovirus (CMV). Colonoscopy showed diffuse polypoid hyperplasia with the absence of rectal involvement. Although the UC was in remission from steroid or total enteral nutrition, but the anemia is getting worse. The Coombs test showed negative, who is subsequently diagnosed by warm autoantibodies of IgA type and treated with mycophenolate mofetil (MMF) 2g daily. Both UC and AIHA were improved from treatment.

Conclusion: AlHA can be secondary to UC, infection, or drugs, in which IgA autoantibody AlHA is a special situation. When gastroenterologist and Immunologist, and hematologist encounter patients with "negative" Coombs test acquired hemolytic anemia, AlHA should be considered in the differential diagnosis. MMF may be an option for the treatment of UC patients with AlHA.

\section{Background}

Ulcerative colitis (UC) is a chronic inflammation disease of colon with unknown etiology, in which the rectum is often the most severely inflamed region of the colon. However, the distal rectum may appear endoscopically normal in $5 \%$ of newly diagnosed adult patients, which is called rectal sparing ${ }^{1}$. Cytomegalovirus (CMV) lesions may be caused by primary infection, reactivation of latent virus, or reinfected via transplantation/blood transfusion. CMV lesions included pneumonia, retinitis, and colitis. Previous epidemiological data regarding inflammatory bowel disease (IBD) coexistent with CMV infection revealed that patients with UC have a higher risk of CMV infection than patients with Crohn's disease (CD) (in whom the risk is lower than $5 \%$ ), but its role in exacerbation of UC remains unclear ${ }^{2}$. At present, we believe that CMV can be divided into "culprit", "accomplice" and "passer-by" through the influence of CMV on the disease progression and clinical outcome of UC. It is proposed that whether antiviral therapy should be individualized, antiviral therapy may be administrated in UC patients of steroid-refractory/dependent with high-grade CMV infection ${ }^{3}$.

The most common causes of anemia in UC patients include blood loss, intestinal inflammation, and malabsorption. UC accompanied with autoimmune hemolytic anemia (AIHA) is very rare, and the incidence is about $1-2 \%{ }^{4}$. In addition, the association between AlHA and UC has been poorly described. Some scholars thought AIHA is the extraintestinal manifestation of IBD, and some scholars thought it is an autoimmune disease coexisting with IBD. The onset of AlHA maybe implied that UC is more aggressive $e^{5,6}$. Confirmation of AlHA usually relies on the direct Coombs test, but several condition may relation to the negative of direct Coombs test, hence a negative direct Coombs test can not completely rule out AIHA. In generally, high dose corticosteroid, either alone or in combination with 
immunosuppressant, is the basis of treatment of AlHA. If drug treatment is unsuccessful, splenectomy or even total colectomy can be selected ${ }^{7}$. Here in this paper, we report a case of AlHA associated with steroid-dependent rectal sparing UC who has a negative result of direct Coombs test. The patient was subsequently diagnosed by warm autoantibodies of IgA type and treated efficacy with oral mycophenolate mofetil (MMF). To our knowledge, this is the first case report about treatment of steroiddependent UC associated with severe AlHA with MMF.

\section{Case Presentation}

A 24-year-old male was first admitted to our clinic with massive bloody diarrhea, fatigue, and pale in June 2020. Before admission, the patient was diagnosed with UC complicated with CMV colitis in July 2019 for the similar symptoms in other hospital, withing a positive serum CMV-DNA PCR test and ulcerative lesion of whole colon excepted rectal. Although meselazine had been prescribed, he had given up medications and follow-up visit without a doctor's recommendation 8 months before admission. His hemoglobin (HB) concentration was $3.3 \mathrm{gr} / \mathrm{dl}, \mathrm{MCV}$ was $80.9 \mathrm{fL}$ and albumin was $23.2 \mathrm{~g} / \mathrm{L}$ with a Creactive protein (CRP) level of $40.1 \mathrm{mg} / \mathrm{L}$. One week after emergency blood transfusion and intravenous steroid (hydrocortisone $300 \mathrm{mg} / \mathrm{d}$ ) therapy, the symptoms of bloody stool and general conditions were better than before. However, colonoscopy revealed multiple deep gouge ulcers and spontaneous bleeding in the sigmoid colon, without rectal involvement (Fig. 1). After additional intravenous ganciclovir for antiviral treatment, the patient's stool returned to normal, and he was discharged. Since then, the patient has been taking mesalazine $(4 \mathrm{~g} / \mathrm{d})$, folic acid, mecobalamin and iron regularly, and the stool has been yellow and shaped, once every two days. Meanwhile, the dose of oral steroids was gradually reduced. After prednisone was reduced to $10 \mathrm{mg} /$ day, the patient experienced a decrease in hemoglobin and albumin again. 3 months after discharge, his HB concentration was decreased to $6.3 \mathrm{gr} / \mathrm{dl}$, and the colonoscopy showed multiple ulceration and erosion of colon with the formation of many pseudopolyps, without the involvement of rectal (Fig. 2). Due to inadequate response, total enteral nutrition was added to the therapy and continued for 3 months. During total enteral nutrition treatment, the patient's anemia and hypoproteinemia were corrected, and the stool was still normal. After the nutritious tube of jejunum was removed because of plugging, the patient experienced a decrease in hemoglobin and albumin again. In March 2021, the patient's HB concentration was $3.9 \mathrm{gr} / \mathrm{dl}, \mathrm{MCV}$ was $80.9 \mathrm{fL}$ and albumin was $20.6 \mathrm{~g} / \mathrm{L}$. In addition, the number and percentage of reticulocytes are as high as $269.3 \times 10^{6} / \mathrm{dL}$ and $8.66 \%$. Twice bone marrow puncture both showed erythroid hyperplasia. Direct Coombs tests were negative, the patient was subsequently diagnosed as AlHA by warm autoantibodies of IgA type and treated with mycophenolate mofetil (MMF) $2 \mathrm{~g}$ daily. From then on, the patient has no symptoms, with the reticulocytes, hemoglobin and albumin returned to normal (Fig. 3). Both UC and AlHA were benefited from treatment.

\section{Discussion}


Colonoscopy is the most used and valuable method for the diagnosis of IBD. The most common endoscopic manifestation of $\mathrm{UC}$ is continuous inflammation involving the distal rectum and extending proximally ${ }^{8,9}$. However, the absence of rectal involvement in the endoscopic impression has been noted in fewer than $5 \%$ of adult patients without any topical treatment, known as rectal sparing ${ }^{10}$. In fact, rectal sparing is more common in pediatric patients, patients with fulminant colitis or means the effects of topical and oral therapy. Other studies have found that absolute rectal sparing does not occur in UC patients even after a long-term treatment, patchy histologic inflammation may also be seen in these patients. ${ }^{11}$. In this case, one of our puzzles was that the rectal mucosa was smooth and intact, the vascular texture was clear, which did not conform to the typical endoscopic manifestations of UC. However, it should be noted that some patients after treatment can be manifested as discontinuous lesions due to the inflammation regression in part of the intestinal. This patient had received short-term sulfasalazine plus hormone enema in another hospital. In the later colonoscopy, we also performed multipoint biopsy and found chronic inflammation of rectal mucosa. Meanwhile, twice bone marrow puncture also ruled out the primary diseases of the blood system. Therefore, we believe that the diagnosis of UC is tenable.

An association between UC and CMV infection was first reported in $1961^{12}$. In UC, CMV infection may present with 2 coexisting conditions: $\mathrm{CMV}$ colitis (where $\mathrm{CMV}$ itself causes colitis) or $\mathrm{CMV}$ infection ${ }^{13}$. Some studies have indicated widespread mucosal defect, cobblestone-like appearance, punched out, longitudinal, and irregular ulceration has been suggested as a characteristic colonoscopic finding in patients with UC complicated by CMV infection ${ }^{14,15}$. The gold standard for CMV colitis included histologic analysis of the colonic biopsy specimen performed by hematoxylin and eosin staining, an immunohistochemical study and amplification of CMV DNA by a qualitative and quantitative PCR in colonic tissue. But all the methods identify a colonic infection of MCV have their advantages as well as disadvantages, the positive rates are low. Furthermore, the treatment of antivirus with CMV infection in UC patients remains very controversial. It is proposed that whether antiviral therapy should be individualized, study suggested antiviral therapy may be indicated for patients of steroid-refractory/dependent UC with high-grade or low-grade CMV infection with endoscopically large ulcers ${ }^{3}$. The serum CMV DNA PCR test of this patient was positive 11 months ago, and the patient was markedly improved after antiviral treatment. Although our case did not meet the gold standard for diagnosis of CMV colitis, he had a history of CMV colitis and had risk factors for reactivation. After treatment of steroid, the symptoms only partially improved, and multiple deep ulcers were found by colonoscopy. Therefore, we chose intravenous ganciclovir for antiviral treatment, and the patient's symptoms improved significantly, his stool returned to normal, and he was discharged after improvement.

The confirmation of AlHA usually depends on the detection of erythrocyte membrane-bound autoantibodies using direct Coombs test ${ }^{16}$. However, the negative direct Coombs test does not completely rule out the diagnosis of AlHA. The IgG antibody and/or complement (C3) protein detection method may fail in about $5 \%$ of $\mathrm{AlHA}^{17}$. In rare cases of IgA autoantibody mediated AlHA, the direct Coombs test may be negative because the commonly used polyspecific direct Coombs test reagent 
contains only anti IgG and anticomplement antibodies. In cases suspected of AlHA but with a negative Coombs test, some tests have been used to confirm the antibody sensitization of erythrocytes, such as the complement-fixing, antibody consumption test ${ }^{18,19}$, enzyme-linked anti-lgG assays, flow cytometric analysis, and a variety of augmented sensitivity tests ${ }^{20-24}$. However, most hospitals usually do not carry out these tests, so confirmed diagnosis of AlHA in patients with negative direct Coombs test is difficult. This case presented a severe refractory anemia inconsistent with disease activity of UC. Although the direct Coombs test was negative, the reticulocyte count and percentage were very high, with the presence of warm autoantibodies of IgA type, the final diagnosis of AlHA is established.

The cases of AlHA may be primary or associated with infection, a lymphoproliferative disease, or an autoimmune disease. AlHA accompanied in UC is rare and highly severe. Although the prevalence of AlHA in UC is low, autoimmune etiology should be considered in the pathogenesis of anemia in UC as well ${ }^{6,25}$, 26 . Studies showed the prevalence of AlHA is between $0.2-1.7 \%$ of all UC patients ${ }^{27,28}$, which is noticeably higher than the reported lifetime prevalence in the general population ${ }^{29}$. To date, the association of UC and AlHA has been few reported in case reports. Hemolytic anemia can occur before, with or after the diagnosis of UC, or several years after colectomy. Marrow in such cases often showed erythroid hyperplasia. But the pathogenesis is uncertain, which may be related to cross antigen and nonspecific immunity. Since both conditions are autoimmune and have connections, steroids and immunosuppressants are effective for both diseases. Since the steroids cannot be used for long-term maintenance treatment, we chose mycophenolate mofetil (MMF) for this case, as research shows MMF is effective in AlHA company with autoimmune or lymphoproliferative diseases. AlHA patients showed a complete or good partial response to MMF treatment ${ }^{30}$. MMF has been previously reported in IBD patients, especially those who are steroid dependent, refractory or intolerant to more traditional therapies. Furthermore, MMF is generally well tolerated with few side-effects ${ }^{31}$. This patient was then treated with MMF $2 \mathrm{~g}$ daily. From then on, the patient has no symptoms, with the reticulocytes, hemoglobin and albumin returned to normal. Both UC and AlHA were benefited from treatment. To our knowledge, this is the first case report about treatment of steroid-dependent UC associated with severe AlHA with oral MMF. By inducing remission of steroid-dependent UC, MMF not only saved the young man from colectomy, but also stopped the treatment of conventional drugs, splenectomy resistance or blood transfusion. Our case suggests that MMF may have a beneficial effect in the treatment of another extraintestinal manifestation, AlHA.

In conclusion, the diagnosis of atypical UC is difficult, and some patients may take opportunistic infection as the first manifestation. Rectal exemption in adults is more common in post-treatment or severe patients, and absolute rectal exemption is very rare. Clinically, there are few cases that meet the gold standard of CMV colitis. For patients with poor treatment effect, positive CMV-DNA in serum, or characteristic manifestations such as longitudinal or deep ulcers, early antiviral treatment can improve the prognosis of patients. AlHA can be secondary to UC, infection, or drugs, in which IgA autoantibody AlHA is a special situation. When gastroenterologist and Immunologist, and hematologist encounter 
patients with "negative" Coombs test acquired hemolytic anemia, AlHA should be considered in the differential diagnosis. MMF may be an option for the treatment of UC patients with AlHA.

\section{Abbreviations}

UC: ulcerative colitis; AlHA: Autoimmune hemolytic anemia; CMV: cytomegalovirus; MMF: mycophenolate mofetil; IBD: inflammatory bowel disease; Crohn's disease (CD); HB: hemoglobin; CRP: C-reactive protein.

\section{Declarations}

\section{Ethics approval and consent to participate}

All procedures followed were in accordance with the ethical standards of the responsible committee on human experimentation (Xi'an Jiaotong University, China) and with the Helsinki Declaration of 1975, as revised in 2008(5).

\section{Consent for publication}

The patient has given written consent for the personal or clinical details along with any identifying images to be published in this study.

\section{Availability of data and materials}

The authors can confirm that all relevant data are included in the article.

\section{Competing interests}

The authors declare that they have no competing interests.

\section{Funding}

This study was supported by grants from the medical and health guidance project of Xiamen (No. 3502Z20209156) and the youth program of Shaanxi Natural Science Foundation (No. 2021 JQ-420). The funding bodies had no role in the study design, collection, analysis, or interpretation of the data.

\section{Authors' contributions}


SMS and BX collected and analyzed the data, performed the report, and authored the work. HTS, XJQ, FRC, and JJ assisted with data interpretation, wrote the manuscript, and approved the final version. $\mathrm{XL}$ is the corresponding author. All authors approved the final version of the manuscript.

\section{Acknowledgements}

We would like to acknowledge our patient for his consent to publish this case report.

\section{Author details}

1. Department of Gastroenterology, the Second Affiliated Hospital of Xi'an Jiaotong University, Shannxi Provincial, Key Laboratory of Gastrointestinal Motility Disorders, Shannxi Provincial, Clinical Research Center of Gastrointestinal Diseases, Xi'an, Shaanxi Province, 710004, P.R. China

2. Department of General surgery, the Chenggong Hospital Affiliated to Xiamen University (Central Hospital of the 73th Chinese People's Liberation Army), Xiamen Fujian Province, 361003, P.R. China

\section{References}

1. Turner D, Levine A, Escher JC, et al. Management of pediatric ulcerative colitis: joint ECCO and ESPGHAN evidence-based consensus guidelines. J Pediatr Gastroenterol Nutr 2012;55:340-61.

2. Nakase $H$, Yoshino $T$, Honzawa $Y$, et al. Low prevalence of $C M V$ infection in patients with Crohn's disease in comparison with ulcerative colitis: effect of different immune response on prevalence of CMV infection. Dig Dis Sci 2010;55:1498-9.

3. Pillet S, Pozzetto B, Roblin X. Cytomegalovirus and ulcerative colitis: Place of antiviral therapy. World J Gastroenterol 2016;22:2030-45.

4. Giannadaki E, Potamianos S, Roussomoustakaki M, et al. Autoimmune hemolytic anemia and positive Coombs test associated with ulcerative colitis. Am J Gastroenterol 1997;92:1872-4.

5. Bianco C, Coluccio E, Prati D, et al. Diagnosis and Management of Autoimmune Hemolytic Anemia in Patients with Liver and Bowel Disorders. J Clin Med 2021;10.

6. Uzzan M, Galicier L, Gornet JM, et al. Autoimmune cytopenias associated with inflammatory bowel diseases: Insights from a multicenter retrospective cohort. Dig Liver Dis 2017;49:397-404.

7. Altman AR, Maltz C, Janowitz HD. Autoimmune hemolytic anemia in ulcerative colitis: report of three cases, review of the literature, and evaluation of modes of therapy. Dig Dis Sci 1979;24:282-5.

8. Rubin DT, Ananthakrishnan AN, Siegel CA, et al. ACG Clinical Guideline: Ulcerative Colitis in Adults. Am J Gastroenterol 2019;114:384-413.

9. Ordas I, Eckmann L, Talamini M, et al. Ulcerative colitis. Lancet 2012;380:1606-19. 
10. Glickman JN, Odze RD. Does rectal sparing ever occur in ulcerative colitis? Inflamm Bowel Dis 2008;14 Suppl 2:S166-7.

11. Joo M, Odze RD. Rectal sparing and skip lesions in ulcerative colitis: a comparative study of endoscopic and histologic findings in patients who underwent proctocolectomy. Am J Surg Pathol 2010;34:689-96.

12. Powell RD, Warner NE, Levine RS, et al. Cytomegalic inclusion disease and ulcerative colitis; report of a case in a young adult. Am J Med 1961;30:334-40.

13. Nakase $\mathrm{H}$, Matsumura $\mathrm{K}$, Yoshino T, et al. Systematic review: cytomegalovirus infection in inflammatory bowel disease. J Gastroenterol 2008;43:735-40.

14. Hirayama $Y$, Ando $T$, Hirooka $Y$, et al. Characteristic endoscopic findings and risk factors for cytomegalovirus-associated colitis in patients with active ulcerative colitis. World J Gastrointest Endosc 2016;8:301-9.

15. Suzuki H, Kato J, Kuriyama M, et al. Specific endoscopic features of ulcerative colitis complicated by cytomegalovirus infection. World J Gastroenterol 2010;16:1245-51.

16. Gehrs BC, Friedberg RC. Autoimmune hemolytic anemia. Am J Hematol 2002;69:258-71.

17. Grimaldi D, Limal N, Noizat-Pirenne F, et al. [IgA-mediated auto-immune haemolytic anaemia revealing a hepatitis C virus infection]. Rev Med Interne 2008;29:135-8.

18. Gilliland BC, Baxter E, Evans RS. Red-cell antibodies in acquired hemolytic anemia with negative antiglobulin serum tests. N Engl J Med 1971;285:252-6.

19. Gilliland BC. Coombs-negative immune hemolytic anemia. Semin Hematol 1976;13:267-75.

20. Biagi E, Assali G, Rossi F, et al. A persistent severe autoimmune hemolytic anemia despite apparent direct antiglobulin test negativization. Haematologica 1999;84:1043-5.

21. Chaudhary R, Das SS, Gupta R, et al. Application of flow cytometry in detection of red-cell-bound IgG in Coombs-negative AlHA. Hematology 2006;11:295-300.

22. Fayek MH, Saad AA, Eissa DG, et al. Role of gel test and flow cytometry in diagnosis of Coombs' negative autoimmune haemolytic anaemia. Int J Lab Hematol 2012;34:311-9.

23. Wang Z, Shi J, Zhou Y, et al. Detection of red blood cell-bound immunoglobulin G by flow cytometry and its application in the diagnosis of autoimmune hemolytic anemia. Int $\mathrm{J}$ Hematol 2001;73:18893.

24. Thedsawad A, Taka O, Wanachiwanawin W. Development of flow cytometry for detection and quantitation of red cell bound immunoglobulin $\mathrm{G}$ in autoimmune hemolytic anemia with negative direct Coombs test. Asian Pac J Allergy Immunol 2011;29:364-7.

25. Lakatos L, Pandur T, David G, et al. Association of extraintestinal manifestations of inflammatory bowel disease in a province of western Hungary with disease phenotype: results of a 25-year followup study. World J Gastroenterol 2003;9:2300-7.

26. Gumaste V, Greenstein AJ, Meyers R, et al. Coombs-positive autoimmune hemolytic anemia in ulcerative colitis. Dig Dis Sci 1989;34:1457-61. 
27. Case records of the Massachusetts General Hospital. Weekly clinicopathological exercises. Case 72000. A 23-year-old man with hemolytic anemia and bloody diarrhea. N Engl J Med 2000;342:722-8.

28. Podolsky DK. Inflammatory bowel disease. N Engl J Med 2002;347:417-29.

29. Eaton WW, Rose NR, Kalaydjian A, et al. Epidemiology of autoimmune diseases in Denmark. J Autoimmun 2007;29:1-9.

30. Howard J, Hoffbrand AV, Prentice HG, et al. Mycophenolate mofetil for the treatment of refractory auto-immune haemolytic anaemia and auto-immune thrombocytopenia purpura. $\mathrm{Br} \mathrm{J}$ Haematol 2002;117:712-5.

31. Simmons WD, Rayhill SC, Sollinger HW. Preliminary risk-benefit assessment of mycophenolate mofetil in transplant rejection. Drug Saf 1997;17:75-92.

\section{Figures}
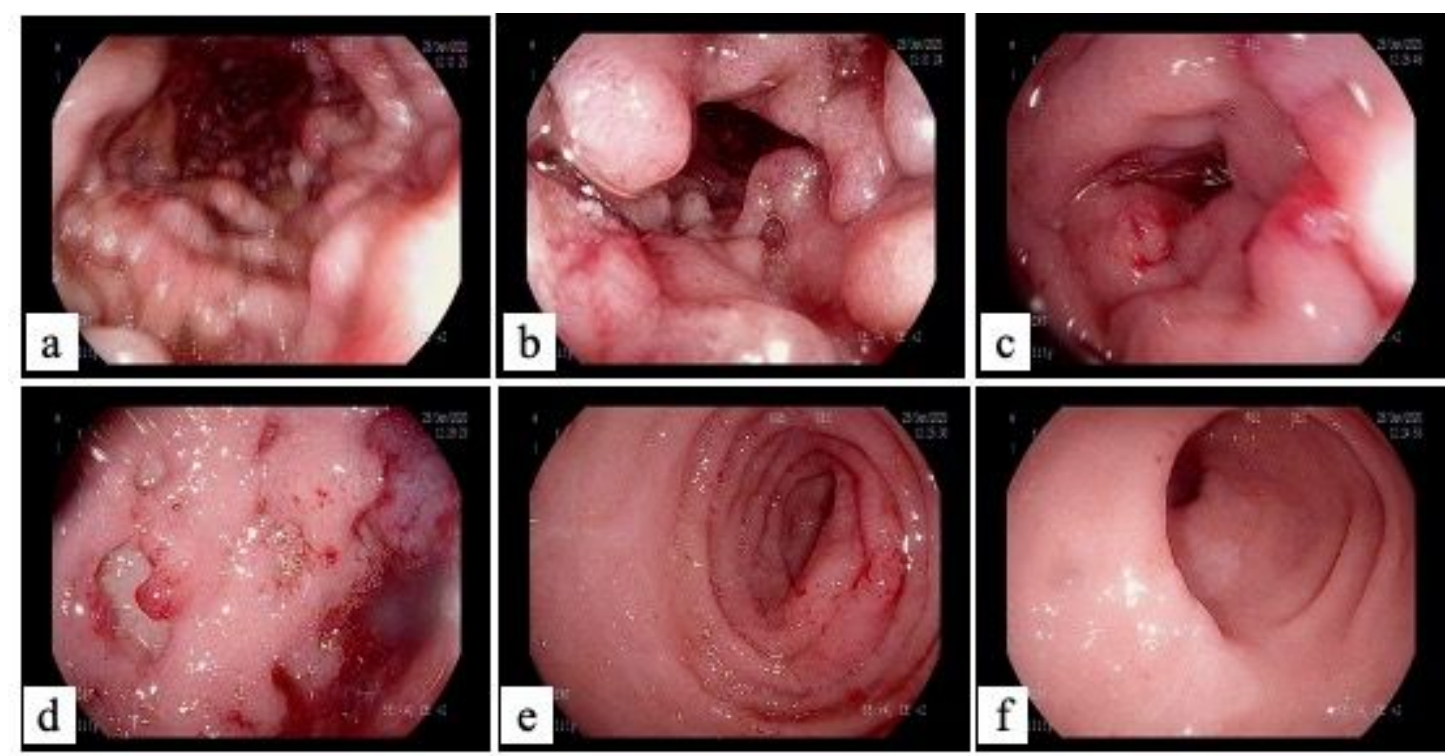

\section{Figure 1}

The colonoscopy (2020-6-28) revealed multiple deep gouge ulcers and spontaneous bleeding in the sigmoid colon, without rectal involvement. 

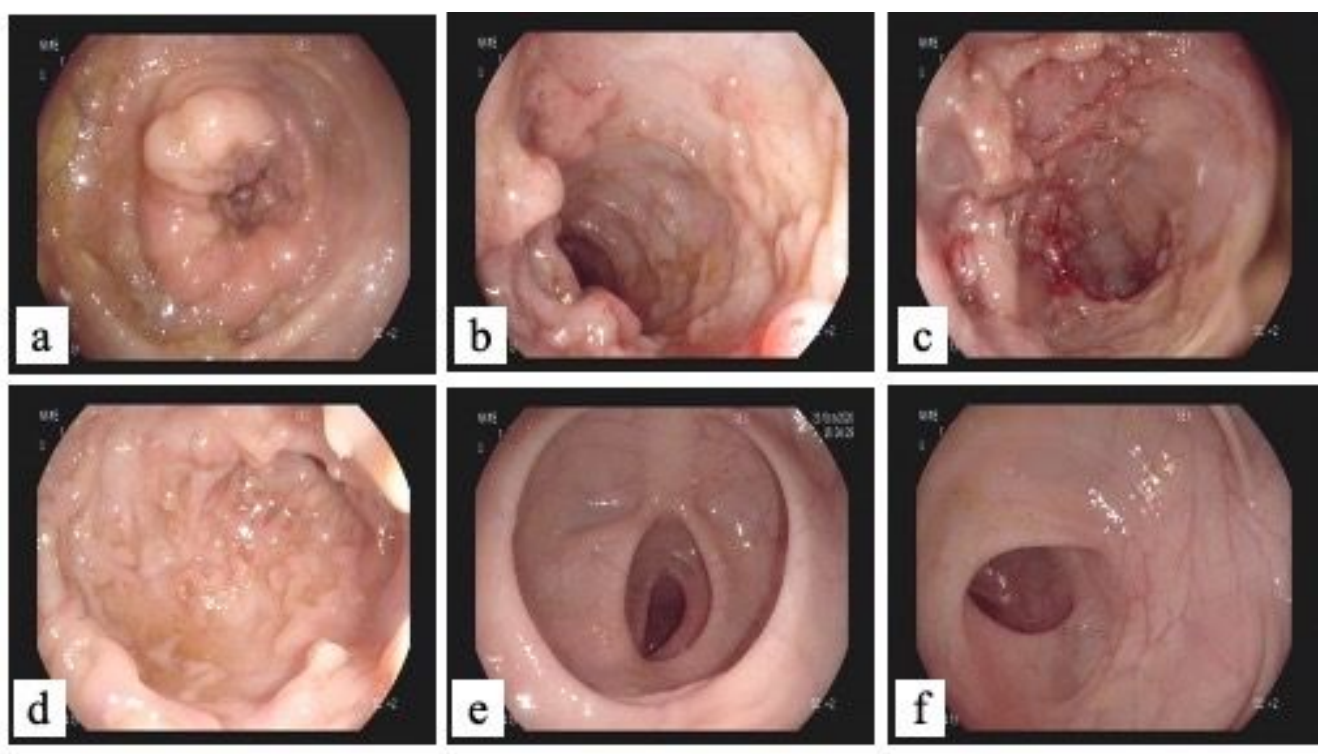

Figure 2

The colonoscopy showed multiple ulceration and erosion of colon with the formation of many pseudopolyps, without the involvement of rectal.

\section{Reticulocyte count $\left(\times 10^{9} / \mathrm{L}\right)$}

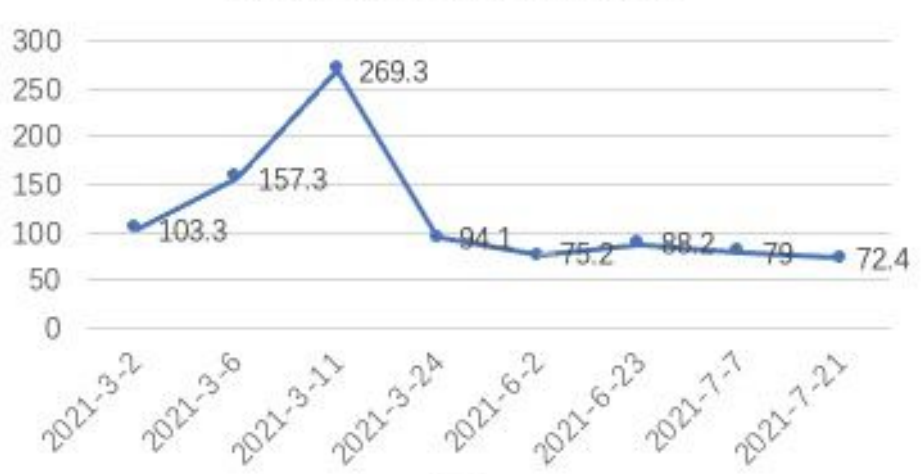

(a)

$\mathrm{HB}(\mathrm{g} / \mathrm{L})$

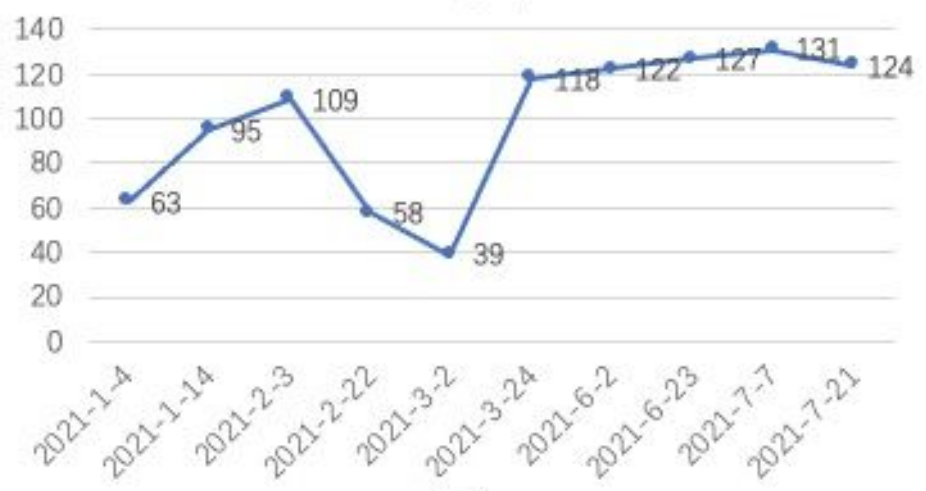

(c)
Reticulocyte percentage (\%)

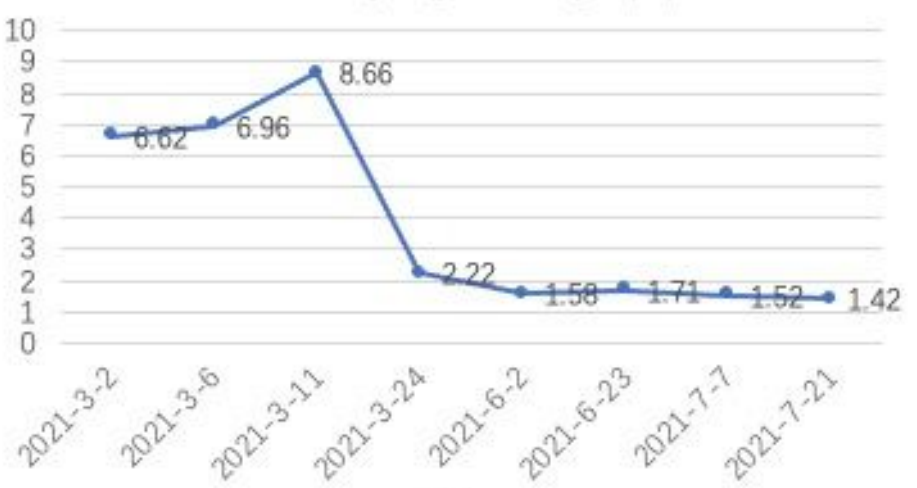

(b)

$\operatorname{ALB}(g / L)$

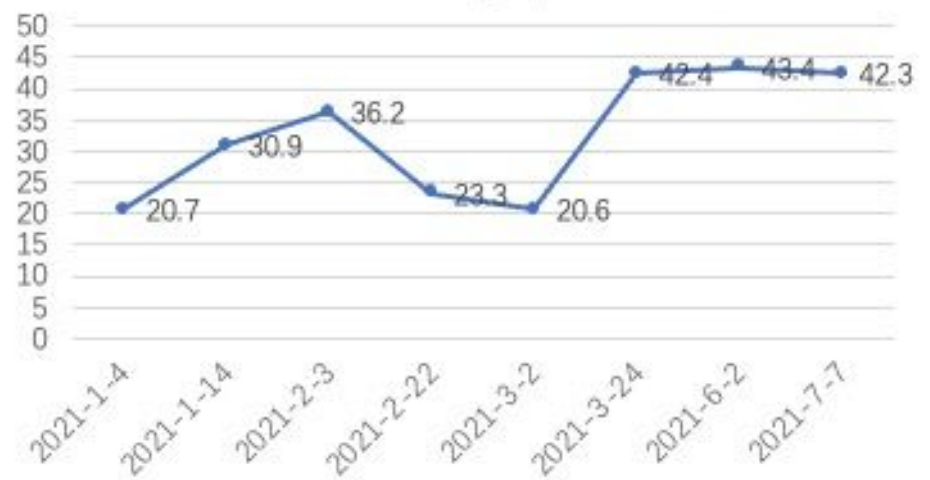

(d)

Figure 3 


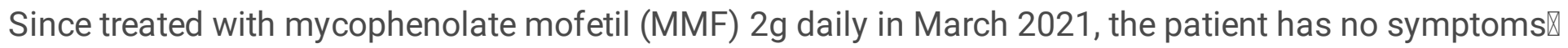
with the reticulocytes $(a, b)$, hemoglobin (c) and albumin (d) returned to normal. 